DOI: $10,4046 / \operatorname{trd} 2011,70,3.224$

ISSN: 1738-3536(Print)/2005-6184(Online)

Tuberc Respir Dis 2011;70:224-234

Copyright(C)2011. The Korean Academy of Tuberculosis and Respiratory Diseases. All rights reserved.

\title{
A Comparative Study of Nursing Home-Acquired Pneumonia with Community-Acquired Pneumonia
}

\author{
Young-Jae Cho, M.D. ${ }^{1,2}$, Bong-Ki Jung, M.D. ${ }^{1}$, Joon-Seok Ahn, M.D., Ph.D. ${ }^{1}$ \\ ${ }^{1}$ Department of Internal Medicine, Kangnam Hospital, Chuncheon, ${ }^{2}$ Division of Pulmonary and Critical Care Medicine, \\ Department of Internal Medicine and Lung Institute, Seoul National University College of Medicine, Seoul, Korea
}

Background: Little data is available regarding hospitalized patients with nursing home-acquired pneumonia (NHAP). This is unfortunate because there is an increasing number of elderly persons who are living in nursing homes in Korea. The aim of this study was to compare clinical characteristics and treatment responses of NHAP with community-acquired pneumonia (CAP).

Methods: Patients with pneumonia who were admitted from eight nursing homes or from their own homes were enrolled between May 2007 and April 2009. Their clinical characteristics and treatment responses were reviewed retrospectively, and differences between the two groups were analyzed.

Results: Of 110 Patients with pneumonia, 66 (60\%) were from nursing homes and their median age was 84. In the NHAP group, functional performance status was significantly poorer, classical symptoms of pneumonia were less severe, and multi-lobe involvement (on chest radiographs) was more frequent than in the CAP group. Patients with NHAP more frequently showed lymphocytopenia, anemia, hypoalbuminemia, hypoxemia, and elevated blood urea nitrogen on admission. The mean CURB-65 score was 2.2 in the NHAP group, higher than 1.7 in the CAP group ( $\mathrm{p}=0.004)$, and multi-drug resistant pathogens were also highly identified in NHAP group (39\% vS. 10\%, $\mathrm{p}=0.036)$. The mean duration of antibiotic therapy was greater for the NHAP (12.6 days) than for the CAP group (6.6 days) ( $\mathrm{p}<0.001)$. The mortality rate was $23 \%$ in NHAP group, which was significantly higher than $5 \%$ in the CAP group $(\mathrm{p}=0.014)$.

Conclusion: NHAP should be more intensively investigated because of the higher frequency of multi-drug resistant pathogens and mortality than the CAP.

Key Words: Nursing Homes; Pneumonia, Community-Acquired

\section{Introduction}

The classification of pneumonia, traditionally categorized as community-acquired pneumonia (CAP) or hospital-acquired pneumonia (HAP) has been changed because the environmental situations out of hospital are becoming more complex to keep pace with "the aging society $^{\prime 1,2}$. In Korea, the average annual increasing rate

Address for correspondence: Young-Jae Cho, M.D.

Division of Pulmonary and Critical Care Medicine, Department of Internal Medicine and Lung Institute, Seoul National University College of Medicine, 101, Daehang-ro, Jongno-gu, Seoul 110-744, Korea

Phone: 82-2-2072-2228, Fax: 82-2-762-9662

E-mail: lungdrcho@gmail.com

Received: Dec. 21, 2010

Accepted: Jan. 20, 2011 of long-term care facilities, represented by nursing home, was remarkable during recent 4 years. Unquestionably, this was caused by the elderly population explosion and the introduction of the National Long-Term Care Insurance. There were more than 2,000 nursing homes in Korea, in which about $1.2 \%$ of the elderly were lived currently. For the same time, the mortality rate of pneumonia in Korea hold the 9th ranks, increased $70 \%$ during the present decade, especially in the elderly people ${ }^{3}$.

Nursing home-acquired pneumonia (NHAP) is probably the largest subgroup of healthcare-associated pneumonia (HCAP), and the number of cases has increased rapidly in recent years ${ }^{4}$. In addition, the elderly patients who were admitted in hospital due to NHAP would be 
held markedly with exceeding medical problems such as unreliable communication, poor social-economical status, absence of familial representatives, etc ${ }^{5,6}$. However, there were extremely little evidences about NHAP, mainly being come from both a new category of respiratory infections and the variety of definitions.

Another big problem of NHAP is the choice treatment for patients with risks of multi-drug resistant (MDR) pathogens. In view of the worldwide threat of increasing resistance to antimicrobial drugs, the acceptance of treating NHAP as the guideline of HAP without relevant evidences would increase the overuse or misuse of antimicrobial drugs, before long produce troublesome pressure in selecting antibiotics for drug-resistant organisms. Therefore, more established data are needed to put the clinical problems of NHAP into perspective ${ }^{7}$.

Herein, we tried to compare the clinical characteristics and treatment responses of patients with pneumonia who were admitted from nursing homes (NHAP group) with those from their own homes (CAP group). To our knowledge, this is the first clinical data about NHAP in Korea.

\section{Materials and Methods}

\section{Study setting and subjects}

This study was conducted at about 200-bed sized, community-based, secondary hospital in Chuncheon, Kangwon-do. Residents of eight nursing homes located at the same city or own homes were enrolled from May 2007 to April 2009, who admitted with pneumonia through emergency department or outpatient clinic. Targeted nursing homes were managed by registered nurses and care workers, and financially supported by municipal or religious organizations. Patients with autoimmune disorder requiring immunosuppressant, current malignancy underwent with chemotherapy or who suffered acquired immune deficiency syndrome were not included. In addition, patients who received antibiotics within 72 hours or transferred from other hospital before enrollment were also excluded. Their demographics; clinical characteristics such as co-morbidities, rou- tine laboratory results, functional status, clinical symptoms and signs, and radiographic features; treatment responses such as duration of antibiotic use or hospital admission, empirical antibiotics regimens, the need of intensive care unit (ICU) management including appliance of mechanical ventilation and successful weaning, in-hospital mortality, and post-discharge follow-up of them were reviewed retrospectively and the differences between NHAP and CAP group were analyzed. In NHAP group, more detailed differences were also compared according to the presence of MDR pathogens. Initially, we included 113 patients with pneumonia aged 20 years and over, however, three patients in CAP group who were under fifty years, which was not matched with NHAP group also were excluded for comparable analysis.

\section{Definitions}

The diagnostic criteria of pneumonia were satisfied with newly detected infiltrates on chest x-ray (CXR) or chest computed tomography (CT) and two or more than clinical findings as follows; 1 ) fever (temperature $\geq$ $38.0^{\circ} \mathrm{C}$ ) or hypothermia (temperature $\leq 35.0^{\circ} \mathrm{C}$ ), 2) leukocytosis or leukopenia, 3) newly developed cough with or without purulent sputum, pleuristic chest pain, or dyspnea, and 4) altered breath sounds on auscultation or hypoxemia in blood gas analysis ${ }^{8,9}$. A radiologist working in the study hospital interpreted all of the radiological findings of CXR or CT and one investigator reviewed all of the radiographic results.

Microbiologically, Gram's stain and culture of sputum with a set of blood culture prior to antibiotics administration were done, when available. An etiology diagnosis was based by results of sputum culture only in cases of the acceptable grade 4 or 5 of Gram's stain, epithelial cells <10/low power field (LPF) or 10 25/LPF with white blood cell (WBC) $>25 / \mathrm{LPF}$.

For the scales of evaluating patient's functional status, the Eastern Cooperative Oncology Group (ECOG) Performance Status criteria were borrowed as same as Lim's study ${ }^{10}$. Originally, these scales were supposed to assess the progression of cancer patient's disease, that is how 
the disease affects the daily living abilities of the patient, and determine appropriate treatment and prognosis. By the ECOG scale, grade 0 means that the patient is fully active, able to carry on all performance without restriction; grade 1 restricted in physically strenuous activity but ambulatory and able to carry out work of a light or sedentary nature; grade 2 ambulatory but unable to carry out any work activities up and about more than $50 \%$ of waking hours, grade 3 capable of only limited self-care, confined to bed or chair more than 50\% of waking hours, grade 4 completely disabled and totally confined to bed or chair, grade 5 dead, respectively ${ }^{11}$.

The confusion, urea nitrogen, respiratory rate, blood pressure (CURB)-65 is a clinical prediction rule that has been validated for predicting mortality in community-acquired pneumonia and the score is an acronym for each of the risk factors measured ${ }^{12}$. Each risk factor scores one point, for a maximum score of 5: Confusion of new onset, blood urea nitrogen $>19 \mathrm{mg} / \mathrm{dL}$, respiratory rate of 30 breaths per minute or greater, blood pressure less than $90 \mathrm{~mm} \mathrm{Hg}$ systolic or diastolic blood pressure 60 $\mathrm{mm} \mathrm{Hg}$ or less, and age 65 or older.

Appropriate antibiotic therapy was defined as the compatible use of empirical or consequent antibiotics directed to the specific microorganism according to susceptibility test criteria for respiratory pathogens with successful clinical response. We also defined the multidrug resistant pathogens as Methicillin-resistant Staphylococcus Aureus (MRSA) or the gram negative pathogen resistant to more than 2 of the following drug classes: 3rd or 4th generation cephalosporins, beta-lactam/beta-lactamase inhibitor combinations, carbapenems, fluoroquinolones, and aminoglycosides.

\section{Statistical analysis}

Statistical comparisons between the two groups were performed using commercial software (SPSS for Windows version 17.0; SPSS Inc., Chicago, IL, USA). Results are expressed as mean values \pm standard deviation (SD) or case numbers/total patients (\%). To detect significant differences between groups, we used the $\chi^{2}$ or Fisher exact test for categorical variables, and the Student's t-test or Mann-Whitney test for continuous variables, whether the variables appropriate parametric or nonparametric method. Statistical significance was assumed at $\mathrm{p}<0.05$, all reported $\mathrm{p}$-values are 2-tailed.

\section{Results}

\section{Baseline characteristics}

Of 110 patients, $66(60 \%)$ patients were admitted from nursing home due to pneumonia. Their median age was 84 years, which was significantly older than that of CAP group. Sex distribution was similar between both groups. Common co-morbidities of the NHAP group were hypertension, dementia, cerebrovascular accident (CVA), and ileus; 46 (69.7\%), 33 (50.0\%), 31 (47.0\%), and $16(24.2 \%)$, in order. These co-morbidities were significantly more frequent than CAP group, however, chronic airway disease and sequelae of tuberculosis were vice versa (Table 1). In NHAP group, the average score of functional status based by ECOG scale was $3.4 \pm 1.3$ compared with $1.4 \pm 0.7$ in CAP group ( $p$ $<0.001$ ). Of laboratory parameters, lymphocytopenia, anemia, hypoalbuminemia, hypoxemia, and elevated blood urea nitrogen (BUN) were significant findings in NHAP group. There were no significant difference in prior treatment of antibiotics within 3 months between both groups (12/66 [18.2\%] in NHAP vs. 5/44 [11.4\%] in CAP; $\mathrm{p}=0.424)$.

\section{Clinical manifestations and radiographic outcomes}

Patients with NHAP complained less frequently classical respiratory symptoms of pneumonia such as cough (26 [39.4\%] vs. 34 [77.3\%]; $\mathrm{p}<0.001)$ and purulent sputum (34 [51.5\%] vs. 32 [72.7\%]; $\mathrm{p}=0.030)$ with statistically significance, whereas the differences of other symptoms were non-specific except macro-aspiration, that means an aspiration identified with the naked eye of patient's caregivers ( 9 [13.6\%] vs. 1 [2.3\%]; $\mathrm{p}=0.048]$. The most common clinical symptoms in NHAP group was fever $(47 / 66,71.2 \%)$, however the frequency of fever between both groups was not significantly different. Abnormal lung sound in physical examination per- 
Table 1. Baseline characteristics of the study subjects

\begin{tabular}{|c|c|c|c|}
\hline & Nursing home group $(n=66)$ & Community group $(\mathrm{n}=44)$ & $p$-value \\
\hline \multicolumn{4}{|l|}{ Demographics } \\
\hline Mean age, median range & $84(53 \sim 100)$ & 77.5 (52 95) & $0.014^{*}$ \\
\hline \multicolumn{4}{|l|}{ Age group, yr } \\
\hline$<65$ & 2/66 (3.0) & 6/44 (13.6) & \multirow{3}{*}{0.035} \\
\hline $65 \sim 79$ & $19 / 66(28.8)$ & $17 / 44(38.6)$ & \\
\hline$>80$ & $45 / 66(68.2)$ & $21 / 44(47.7)$ & \\
\hline Female & 43/66 (65.2) & $22 / 44(50.0)$ & 0.120 \\
\hline \multicolumn{4}{|l|}{ Co-morbidities } \\
\hline Cerebrovascular accident & $31 / 66(47.0)$ & $7 / 44(15.9)$ & $0.001^{*}$ \\
\hline Dementia & $33 / 66(50.0)$ & $2 / 44(4.5)$ & $<0.001^{*}$ \\
\hline Parkinson's disease & $2 / 66(3.0)$ & $0 / 44(0.0)$ & 0.516 \\
\hline Hypertension & $46 / 66(69.7)$ & $21 / 44(47.7)$ & $0.028 *$ \\
\hline Congestive heart failure & $11 / 66(16.7)$ & $5 / 44(10.6)$ & 0.583 \\
\hline Atrial fibrillation & $6 / 66(9.1)$ & $2 / 44(4.5)$ & 0.472 \\
\hline Diabetes & $16 / 66(24.2)$ & $6 / 44(13.6)$ & 0.226 \\
\hline Chronic airway disease & 12/66 (18.2) & $23 / 44$ (52.3) & $<0.001^{*}$ \\
\hline Sequelae of tuberculosis & $4 / 66(6.1)$ & $11 / 44(25.0)$ & $0.009^{*}$ \\
\hline Chronic liver disease & $2 / 66(3.0)$ & $2 / 44(4.3)$ & 1.000 \\
\hline Chronic kidney disease & $5 / 66(7.6)$ & $1 / 44(2.3)$ & 0.399 \\
\hline Cancer & $1 / 66(1.5)$ & $2 / 44(4.6)$ & 0.297 \\
\hline Use of immunosuppressant & $3 / 66(4.5)$ & $0 / 44(0.0)$ & 0.273 \\
\hline Pressure ulcer & $8 / 66(12.1)$ & $1 / 44(2.3)$ & 0.083 \\
\hline lleus & $16 / 66(24.2)$ & $4 / 44(9.1)$ & $0.048^{*}$ \\
\hline Prior antibiotics, $<3 \mathrm{mo}$ & $12 / 66(18.2)$ & $5 / 44(11.4)$ & 0.424 \\
\hline \multicolumn{4}{|l|}{ Laboratory findings } \\
\hline White blood cell, $/ \mathrm{mm}^{3}$ & $11,469.7 \pm 6,058.2$ & $11,325.0 \pm 6,650.4$ & 0.906 \\
\hline Neutrophil & $9,492.7 \pm 5,747.6$ & $8,742.9 \pm 5,350.5$ & 0.492 \\
\hline Lymphocyte & $993.8 \pm 562.1$ & $1,500.9 \pm 1,449.9$ & $0.012^{*}$ \\
\hline Hemoglobin, g/dL & $10.5 \pm 1.8$ & $11.6 \pm 1.5$ & $0.001^{*}$ \\
\hline Albumin, $\mathrm{g} / \mathrm{dL}$ & $3.2 \pm 0.6$ & $3.5 \pm 0.4$ & $0.004^{*}$ \\
\hline $\mathrm{ALT}, \mathrm{IU} / \mathrm{L}$ & $22.5 \pm 27.1$ & $25.8 \pm 42.6$ & 0.623 \\
\hline BUN, mg/dL & $24.6 \pm 13.1$ & $18.7 \pm 9.1$ & $0.007^{*}$ \\
\hline Creatinine, mg/dL & $1.0 \pm 0.6$ & $1.0 \pm 0.4$ & 0.932 \\
\hline C-Reactive Protein, mg/dL & $113.0 \pm 76.1^{\dagger}$ & $95.5 \pm 65.1$ & 0.215 \\
\hline $\mathrm{Na}, \mathrm{mmol} / \mathrm{L}$ & $136.6 \pm 7.8$ & $137.7 \pm 5.8$ & 0.421 \\
\hline $\mathrm{K}, \mathrm{mmol} / \mathrm{L}$ & $3.9 \pm 0.7$ & $4.0 \pm 0.6$ & 0.227 \\
\hline \multicolumn{4}{|l|}{ Arterial blood gas ${ }^{\ddagger}$} \\
\hline $\mathrm{pH}$ & $7.5 \pm 0.1$ & $7.5 \pm 0.6$ & 0.465 \\
\hline $\mathrm{PaCO}_{2}, \mathrm{~mm} \mathrm{Hg}$ & $32.8 \pm 6.3$ & $33.5 \pm 7.6$ & 0.611 \\
\hline $\mathrm{PaO}_{2}, \mathrm{~mm} \mathrm{Hg}$ & $64.0 \pm 17.6$ & $72.6 \pm 20.0$ & $0.031^{*}$ \\
\hline $\mathrm{SaO}_{2}, \%$ & $91.1 \pm 5.0$ & $93.7 \pm 3.4$ & $0.003^{*}$ \\
\hline \multicolumn{4}{|l|}{ Functional status } \\
\hline ECOG scale & $3.4 \pm 1.3$ & $1.4 \pm 0.7$ & $<0.001^{*}$ \\
\hline Not limited active & $0 / 66(0.0)$ & $0 / 44(0.0)$ & \\
\hline Limited activity & $2 / 66(3.0)$ & $32 / 44(72.7)$ & \\
\hline Ambulatory >50\% & 10/66 (15.2) & $7 / 44(15.9)$ & \\
\hline Ambulatory <50\% & $24 / 66(36.4)$ & $5 / 44(11.4)$ & \\
\hline Bedridden & $30 / 66(45.5)$ & $0 / 44(0.0)$ & \\
\hline Levin tube or gastrostomy & $5 / 66(7.6)$ & $0 / 44(0.0)$ & 0.082 \\
\hline Foley catheter & $4 / 66(6.1)$ & $0 / 44(0.0)$ & 0.148 \\
\hline Swallowing difficulty & 9/66 (13.6) & $1 / 44(2.3)$ & $0.048^{*}$ \\
\hline
\end{tabular}

Data are presented as numbers/total patients (\%) or mean \pm standard deviation unless otherwise indicated. ${ }^{*} \mathrm{p}<0.05,{ }^{\dagger}$ One patient missing, ${ }^{\ddagger} 63$ patients in Nursing home group and 34 in Community group.

ECOG: Eastern Cooperative Oncology Group; ALT: alanine aminotransferase; BUN: blood urea nitrogen. 
YJ Cho et al: Comparative study of nursing home-acquired pneumonia

Table 2. Clinical manifestations and Radiographic outcomes of pneumonia

\begin{tabular}{|c|c|c|c|}
\hline & Nursing home group $(\mathrm{n}=66)$ & Community group $(n=44)$ & p-value \\
\hline \multicolumn{4}{|l|}{ Clinical symptoms } \\
\hline \multicolumn{4}{|l|}{ Respiratory } \\
\hline Cough & 26/66 (39.4) & $34 / 44(77.3)$ & $<0.001^{*}$ \\
\hline Sputum & $34 / 66(51.5)$ & $32 / 44(72.7)$ & $0.030^{*}$ \\
\hline Dyspnea & 22/66 (33.3) & $20 / 44(45.5)$ & 0.232 \\
\hline Pleuritic pain & $1 / 66(1.5)$ & $4 / 44(9.1)$ & 0.155 \\
\hline Hemoptysis & $0 / 66(0.0)$ & $1 / 44(2.3)$ & 0.400 \\
\hline \multicolumn{4}{|l|}{ Non-respiratory } \\
\hline Fever & 47/66 (71.2) & 23/44 (52.3) & 0.068 \\
\hline Chill/Myalgia & $4 / 66(6.1)$ & $8 / 44(18.2)$ & 0.062 \\
\hline Altered mentality & $4 / 66(6.1)$ & $1 / 44(2.3)$ & 0.646 \\
\hline Macro-aspiration $^{\dagger}$ & $9 / 66(13.6)$ & $1 / 44(2.3)$ & $0.048^{*}$ \\
\hline Uncommon & 16/66 (24.2) & 8/44 (18.2) & 0.490 \\
\hline Abnormal lung sounds & $38 / 66(57.6)$ & $32 / 44(72.7)$ & 0.525 \\
\hline \multirow{2}{*}{\multicolumn{4}{|c|}{$\begin{array}{l}\text { Radiographic features } \\
\text { Involvement of location }\end{array}$}} \\
\hline & & & \\
\hline $\mathrm{RU}+\mathrm{RM}$ & 20/66 (30.3) & $16 / 44(36.4)$ & 0.539 \\
\hline $\mathrm{RL}$ & $52 / 66(78.8)$ & $27 / 44(61.4)$ & 0.054 \\
\hline LU & $7 / 66(10.6)$ & $2 / 44(4.5)$ & 0.311 \\
\hline LL & 42/66 (63.6) & $13 / 44(29.5)$ & $0.001^{*}$ \\
\hline Multi-lobes involvement & $46 / 66(69.7)$ & $13 / 44(29.5)$ & $<0.001^{\star}$ \\
\hline One & $20(30.3)$ & $31(70.5)$ & \\
\hline Two & $37(56.1)$ & $12(27.3)$ & \\
\hline Three & $9(13.6)$ & $1(2.3)$ & \\
\hline Pleural effusions & $22 / 66(33.3)$ & 6/44 (13.6) & 0.095 \\
\hline CURB-65 ${ }^{\ddagger}$ & $2.2 \pm 0.9$ & $1.7 \pm 1.0$ & $0.004^{\star}$ \\
\hline
\end{tabular}

Data are presented as numbers/total patients (\%) or means \pm standard deviation.

${ }^{*} \mathrm{p}<0.05,{ }^{\dagger}$ Aspiration identified with the naked eye of patient's caregivers, ${ }^{\dagger}$ Confusion of new onset; Blood Urea Nitrogen, $>19$ $\mathrm{mg} / \mathrm{dL}$; Respiratory rate of 30 breaths per minute or greater; Blood pressure less than $90 \mathrm{~mm} \mathrm{Hg}$ systolic or diastolic blood pressure $60 \mathrm{~mm} \mathrm{Hg}$ or less, and age 65 years or older.

$\mathrm{RU}$ : right upper; RM: right middle; RL: right lower; LU: left upper; LL: left lower; CURB: confusion, urea nitrogen, respiratory rate, blood pressure.

formed by the investigator also was not significantly different between both groups (38 [57.6\%] vs. 32 [72.7\%]; $\mathrm{p}=0.525$ ). On chest radiographs, the most commonly involved lobe of the lung in NHAP group was right lower field (52/66, 78.8\%), however, the statistically significant difference between both groups was found in left lower field of the lung ( 42 [63.6\%] vs. 13 [29.5\%]; $p<0.001$ ). In addition, multi-lobe (two or more than) involvement of the lung due to pneumonia was also more frequent in NHAP group (46 [69.7\%] vs. 13 (29.5\%); p<0.001). The CURB-65 score was significantly higher in NHAP group $(2.2 \pm 0.9$ vs. $1.7 \pm 1.0 ; \mathrm{p}=0.004)$. Other details were described in Table 2.

\section{Microbiological results}

Table 3 showed the microbiological results of both groups. Not all of the pneumonia was confirmed microbiologically because of poor expectoration or inappropriate group-categorized Gram's stain of sputum. Of all, 44 (40\%) patients were diagnosed with microbiologically confirmed pneumonia, 23 in NHAP group and 21 in CAP group. The most common pathogen was Streptococcus species (9/23 [39.1\%] in NHAP vs. 12/21 [66.6\%] in CAP) and overall distribution of microorganisms between both groups was not significantly different $(\mathrm{p}=0.263$ ). However, MDR pathogens were significantly more frequent in patients with NHAP (9 
Table 3. Microbiological results of pneumonia

\begin{tabular}{|c|c|c|c|}
\hline & Nursing home group $(n=66)$ & Community group $(n=44)$ & p-value \\
\hline Acceptable sputum & 23/66 (34.8) & 21/44 (47.8) & 0.380 \\
\hline Microbiological features & & & 0.263 \\
\hline Streptococcus species & 9/23 (39.1) & $14 / 21$ (66.6) & \\
\hline Acinetobacter baumannii & $2 / 23(8.7)$ & $1 / 21(4.8)$ & \\
\hline Pseudomonas aeruginosa & $3 / 23(13.0)$ & $0 / 21(0.0)$ & \\
\hline MRSA & $3 / 23(13.0)$ & $0 / 21(0.0)$ & \\
\hline Klebsiella pneumoniae & $2 / 23(8.7)$ & $1 / 21(4.8)$ & \\
\hline Escherichia coli & $2 / 23(8.7)$ & $1 / 21(4.8)$ & \\
\hline Enterobacter/Enterococcus & $1 / 23(4.3)$ & $2 / 21(9.5)$ & \\
\hline Others & $1 / 23(4.3)$ & $2 / 21(9.5)$ & \\
\hline Multidrug resistance & 9/23 (39.1) & $2 / 21(9.5)$ & $0.036^{*}$ \\
\hline MRSA & 3 & - & \\
\hline Acinetobacter baumannii & 1 & - & \\
\hline Pseudomonas aeruginosa & 2 & - & \\
\hline Klebsiella pneumoniae & 1 & 1 & \\
\hline Enterobacter cloacae & 1 & - & \\
\hline Others $^{\dagger}$ & 1 & 1 & \\
\hline
\end{tabular}

Data are presented as numbers/total patients (\%).

${ }^{*} \mathrm{p}<0.05,{ }^{\dagger}$ Stenotrophomonas maltophilia, Burkholderia cepacia.

MRSA: Methicillin-resistant Staphylococcus aureus.

Table 4. Subgroup analysis according to Multi-drug resistance (MDR) pathogen

\begin{tabular}{lccc}
\hline \multicolumn{1}{c}{ In NHAP group } & MDR $(\mathrm{n}=9)$ & Non-MDR $(\mathrm{n}=14)$ & p-value \\
\hline Age & $81.4 \pm 10.3$ & $84.1 \pm 5.9$ & 0.507 \\
Sex & $6 / 9(66.7)$ & $11 / 14(78.6)$ & 0.643 \\
CVA & $4 / 9(44.4)$ & $5 / 14(35.7)$ & 1.000 \\
Prior antibiotics, $<3$ mo & $3 / 9(33.3)$ & $0 / 14(0.0)$ & $0.047^{\star}$ \\
Lymphocyte & $1,157.5 \pm 314.5$ & $1,071.7 \pm 719.8$ & 0.284 \\
BUN & $25.6 \pm 19.6$ & $22.5 \pm 11.1$ & 0.850 \\
Albumin & $3.0 \pm 0.7$ & $3.4 \pm 0.7$ & 0.219 \\
PaO, & $66.3 \pm 19.8$ & $64.1 \pm 22.6$ & 0.728 \\
ECOG scale & $3.7 \pm 0.7$ & $3.1 \pm 0.9$ & 0.121 \\
Swallowing difficulty & $3 / 9(33.3)$ & $0 / 14(0.0)$ & $0.047^{\star}$ \\
Multi-lobes involvement & $8 / 9(88.9)$ & $11 / 14(78.6)$ & 0.771 \\
Appropriate antibiotic therapy & $5 / 9(55.6)$ & $8 / 14(57.1)$ & 1.000 \\
Total duration of antibiotics & $16.6 \pm 8.5$ & $18.4 \pm 12.1$ & 0.824 \\
Mechanical ventilated & $2 / 9(22.2)$ & $1 / 14(7.1)$ & 0.538 \\
In hospital mortality & $5 / 9(55.6)$ & $4 / 14(28.6)$ & 0.383 \\
CURB-65 & $2.0 \pm 0.9$ & $2.1 \pm 0.9$ & 0.810 \\
\hline
\end{tabular}

Data are presented as number/total patients (\%) or mean \pm standad deviation. ${ }^{*} p<0.05$.

NHAP: nursing home-acquired pneumonia; CVA: cerebrovascular accident; BUN: blood urea nitrogen; ECOG: Eastern Cooperative Oncology Group; CURB: confusion, urea nitrogen, respiratory rate, blood pressure.

[39.1\%] vs. 2 [9.5\%]; p=0.036). The detailed MDR pathogens were showed in Table 3. In sub-group analysis (Table 4), MDR pathogens in NHAP group were found more frequently in cases of prior treatment of antibiotics within three months or presence of swallowing difficulty (33.3\% vs. 0\%; $\mathrm{p}=0.047$ in both). 
YJ Cho et al: Comparative study of nursing home-acquired pneumonia

\section{Treatment courses and responses (Table 5)}

The total duration of treatment with intravenous antibiotics in hospital was $12.6 \pm 8.8$ days in NHAP group vs. 6.6 \pm 3.2 days in CAP group $(\mathrm{p}<0.001)$. The most common initial regimen of antibiotics was the combination of 3rd cephalosporin plus fluoroquinolone (30/66, $45.5 \%)$ in NHAP group vs. 3rd cephalosporin plus macrolide (20/44, 45.5\%) in CAP group and the distribution of antimicrobial choice was not significantly different between both groups. In NHAP group, more patients were treated with additional antibiotics covering anaerobes without significant difference (11 [16.7\%] vs. 3 [6.8\%]; $\mathrm{p}=0.154$ ), however, less could be treated with appropriate antibiotic therapy with significant difference (39 [59.1\%] vs. 40 [90.0\%]; $\mathrm{p}=0.001$ ). In addition, 37
(56.1\%) patients with NHAP had to need supportive managements in ICU on admission day, and 10 (15.2\%) eventually had been ventilated mechanically. As unexpected result, $90 \%$ of them were weaned from the mechanical ventilation successfully. Total duration of hospitalization in NHAP group was $14.4 \pm 10.6$ days, significantly longer than in CAP group $(7.3 \pm 3.4$ days $)$ and in-hospital mortality was also higher in NHAP group (15 [22.7\%] vs. 2 [4.3\%], p=0.007). There were more patients with high CURB-65 score in NHAP group; however, the trend of mortality according to CURB-65 score did not showed significant difference within each group (Table 6).

Table 5. Treatment courses and Responses of antibiotics

\begin{tabular}{|c|c|c|c|}
\hline & Nursing home group $(n=66)$ & Community group $(n=44)$ & p-value \\
\hline \multicolumn{4}{|l|}{ Mean duration of medication (day) } \\
\hline Intravenous & $12.6 \pm 8.8$ & $6.6 \pm 3.2$ & $<0.001^{*}$ \\
\hline Total & $17.4 \pm 8.9$ & $12.2 \pm 3.5$ & $<0.001^{*}$ \\
\hline Regimens & & & 0.261 \\
\hline 3rd cephalosporin + Macrolide & $14 / 66(21.2)$ & $20 / 44(45.5)$ & \\
\hline Quinolone only & $12 / 66(18.2)$ & $8 / 44(18.2)$ & \\
\hline 3rd cephalosporin + Quinolone & $30 / 66(45.5)$ & $12 / 44(27.3)$ & \\
\hline Piperacillin +Tazobactam & $6 / 66(9.1)$ & $2 / 44(4.5)$ & \\
\hline Imipenem/Meropenem & $1 / 66(1.5)$ & $0 / 44(0.0)$ & \\
\hline Others & $3 / 66(4.5)$ & $2 / 44(4.5)$ & \\
\hline Coverage of anaerobe & $11 / 66(16.7)$ & $3 / 44(6.8)$ & 0.154 \\
\hline Appropriate antibiotic therapy & $39 / 66(59.1)$ & 40/44 (90.9) & $0.001^{*}$ \\
\hline Combined systemic steroid & $26 / 66(39.4)$ & $21 / 44(47.7)$ & 0.502 \\
\hline \multicolumn{4}{|l|}{ Need of Intensive Care Unit } \\
\hline Initial & $37 / 66(56.1)$ & $13 / 44(29.5)$ & $0.007^{\star}$ \\
\hline Transferred & $2 / 66(3.0)$ & $1 / 44(2.3)$ & 1.000 \\
\hline Mechanical ventilated & $10 / 66(15.2)$ & $0 / 44(0.0)$ & $0.006^{*}$ \\
\hline Tracheostomy & $3 / 10$ & - & \\
\hline Weaning & $9 / 10$ & - & \\
\hline \multicolumn{4}{|l|}{ Treatment Outcome } \\
\hline Duration of hospitalization (day) & $14.4 \pm 10.6$ & $7.3 \pm 3.4$ & $<0.001^{*}$ \\
\hline In hospital mortality & $15 / 66(22.7)$ & $2 / 44(4.5)$ & $0.014^{*}$ \\
\hline Respiratory failure & 9 & 1 & \\
\hline Shock/multiorgan failure & 5 & 1 & \\
\hline Congestive heart failure & 1 & - & \\
\hline \multicolumn{4}{|l|}{ Post-discharge follow-up } \\
\hline No readmission within one month & $48 / 51(94.1)$ & $38 / 42(90.5)$ & 0.697 \\
\hline Live after three months & $21 / 44(47.7)$ & $27 / 30(90.0)$ & $0.001^{*}$ \\
\hline
\end{tabular}

Data are presented as number/total patients (\%) or mean \pm standard deviation. ${ }^{*} \mathrm{p}<0.05$. 
Table 6. In hospital mortality according to CURB-65 score

\begin{tabular}{|c|c|c|c|c|c|c|}
\hline & \multicolumn{5}{|c|}{ CURB-65 score } & \multirow{2}{*}{ p-value } \\
\hline & 0 & 1 & 2 & 3 & 4 & \\
\hline $\mathrm{NHAP}, \mathrm{n}=66$ & - & $15(22.7)$ & $29(43.9)$ & $18(27.3)$ & $4(6.1)$ & \\
\hline Mortality & - & $5(33.3)$ & $7(24.1)$ & $2(11.1)$ & $1(25.0)$ & 0.496 \\
\hline CAP, $n=44$ & $3(6.8)$ & 19 (43.2) & $14(31.8)$ & $6(13.6)$ & $2(4.5)$ & \\
\hline Mortality & $0(0.0)$ & $1(5.3)$ & $1(7.1)$ & $0(0.0)$ & $0(0.0)$ & 0.943 \\
\hline Total, $n=110$ & $3(2.7)$ & $34(30.9)$ & $43(39.1)$ & $24(21.8)$ & $6(5.5)$ & \\
\hline Mortality & $0(0.0)$ & $6(17.6)$ & $8(18.6)$ & $2(8.3)$ & $1(16.7)$ & 0.747 \\
\hline
\end{tabular}

Data are presented as number/total patients (\%) or mean \pm standard deviation.

CURB: confusion, urea nitrogen, respiratory rate, blood pressure; NHAP: nursing home-acquired pneumonia; CAP: community-acquired pneumonia.

\section{Discussion}

Though regarded to limited data, our study elucidated the first and unique characteristics of NHAP in Korea. In summary, patients with NHAP had more common co-morbidities in hypertension, dementia, CVA, and ileus; showed poor functional status with swallowing difficulty; were identified with lymphocytopenia, anemia, hypoalbuminemia, elevated blood urea nitrogen, and hypoxemia; presented less classical respiratory symptoms and involved with more lobes of the lung; manifested multi-drug resistant pathogens more predominantly; were hospitalized with longer duration and poor prognosis, compared to those with CAP.

Recently, Yoon et al. ${ }^{13}$ reported clinical and microbial characteristics of HCAP compared with HAP in their tertiary hospital experiences. HCAP in their study showed similar characteristics with HAP, but limitation should be considered that their population was comprised of mixed groups such as long term care hospital, traditional medicine hospital, psychiatric hospital, and nursing home. In contrary, our study population was comprised of relatively homogenous groups directly from nursing home or community. Indeed, our results are closely similar to the first prospective comparative study $^{10}$ of NHAP with CAP in the United Kingdom and recent 10-year observational study ${ }^{5}$ of NHAP, as the matter of clinical symptoms or signs, functional statuses, microbiological results, and mortalities. Considering that informational limitation and selection bias of studies dealing with HCAP in tertiary hospital, our clinical presentations and outcomes about NHAP in Korea would be paid attention to beyond expectation despite of the secondary hospital setting.

Most of all, the results of less frequent classical presentations of NHAP in this study are important to detect pneumonia at the early stage in nursing home residents. Not uncommonly, patient with NHAP would remain asymptomatic or only febrile before diagnosis ${ }^{14}$, although exactly not as same as our results. Johnson et al. ${ }^{15}$ found that nonspecific symptoms were common in the presentation of pneumonia in elderly people, which could apply to patients with NHAP. In addition, patients with NHAP suffered swallowing difficulty at nursing home or macro-aspiration before visiting hospital more frequently as our results. The preservation of swallow function and cough reflex is important defense mechanisms against oropharyngeal aspiration, with abnormalities of both increasing the risk of aspiration pneumonia. While the etiology of aspiration pneumonia is multi-factorial, there is a strong association between dysphagia and the development of aspiration pneumonia in the elderly ${ }^{16}$. Additionally, only aging per se would not be held responsible to increase the risk of aspiration. The incidence of cognitive or neurologic diseases increase with aging and these disorders are strongly associated with impaired swallow function and decreased cough reflex, which resulted in the increased 
risk of aspiration. That's why kinds of protocol assessing and managing dysphagia at nursing home should be needed ${ }^{17,18}$.

It should be focused that MDR pathogens was indentified more frequently in patients with NHAP. There are many reports supporting that microbiological results of NHAP is similar with $\mathrm{HAP}^{5,7,8,10,14,17,19}$, including recent one Korean study as mentioned ${ }^{13}$. The problem is how to choosing the empirical antibiotic regimens to treat NHAP as CAP or HAP, however, established clinical trials are still unavailable. There are two aspects prior to think considering the treatment of NHAP; one is the place to treatment, in nursing home in situ or in hospital which patients are admitted, and the other is how to deal with MDR pathogens ${ }^{20}$. Although the former is out of our discussion, the latter is still debating problems because of many conflict evidences between several studies or expert's opinions. Until now, the American Thoracic Society/Infectious Diseases Society of America (ATS/IDSA) guideline ${ }^{4}$ currently assumes that nursing home residence by itself is one of the major risk factors for MDR organisms; therefore, the recommendation of empirical treatment focuses on these pathogens. However, there is no confident evidence that the broad-spectrum regimens recommended by the ATS/IDSA guideline could be more efficacious compared with the regimens recommended by other CAP guidelines for treating NHAP requiring hospitalization. This is why as follows: basically, these concerns are originated from that the ATD/IDSA guideline is based exclusively on the findings of intubated patients in the ICU setting; additionally, patients who were admitted from nursing home are at a higher risk of upper airway colonization by Gram-negative bacteria or MRSA because of their impaired functional status, which possibly explains a high rate of Gram-negative bacteria or MRSA identified in sputum samples, occurring an overestimation of Gram-negative bacteria or MRSA as true pathogens in $\mathrm{NHAP}^{6}$.

Besides microbiological issues, it should be considered that severe elderly patients with NHAP are more likely to suffer from CVA or dementia, to present with delirium and malnutrition, to have a lower functional status, or to be bed-ridden when compared with simple elderly patients with CAP. Such co-morbidities have all been associated with a higher mortality. Indeed, in recent studies of older patients with NHAP and CAP, patients with NHAP have more severity scores such as the pneumonia severity index, and accordingly mortality remains higher for $\mathrm{NHAP}^{21}$. Frankly, in the real world, we cannot but consider the term "end-of-life pneumonia" that affects the elderly who are left (to die) in nursing homes.

For these several reasons, it is convincing that NHAP should not always be treated the same as $\mathrm{HAP}^{22}$. There were patients who might be treated with too broad spectrum of antibiotics unnecessarily if this approach were used routinely. In reality, whether patients from nursing home treated in the hospital or in the nursing home, many studies have also demonstrated the efficacy of monotherapy regimens, such as fluoroquinolone or cefepime that would not be recommended for patients with HAP at risk for MDR pathogens ${ }^{23}$. In our study, MDR pathogens were more frequently in NHAP group in cases of prior treatment of antibiotics within three months or swallowing difficulty. This result was relatively compatible to other study indicating that risk factors of drug resistant bacteria in severe NHAP was prior antibiotic therapy in the past 3 to 6 months and poor functional status $^{24}$. Based on these evidences, patients with NHAP would need to be divided into each subgroups by evaluating the risk of MDR pathogens ${ }^{22}$. After all, more randomized controlled studies for NHAP should be needed.

Several important limitations of this study should be noted. Firstly, we could not get lower respiratory tract specimens from patients by invasive technique such as bronchoscopy and employ non-cultured methods (eg, serologies, urinary antigen tests, or polymerase chain reaction techniques) to establish the etiologic diagnosis of pneumonia. Therefore, we may have missed other microbiological results of pneumonia due to either early antibiotic administration or inadequate specimens submitted for microbiologic evaluation. As same reason,

\section{2}


our findings could not be applicable to patients with atypical pathogens or viruses. Secondly, although we tried to apply the severity-of-illness score by CURB-65, the result of mortality related to CURB-65 score could not show statistically significant trends in both groups. First of all, small number of study population, especially in higher subgroup of CURB-65 score, probably could cause this limitation. In addition, the CURB-65 would not be appropriate to apply for prediction of mortality in NHAP so far as we allow that NHAP has to be distinct from CAP.

In conclusion, NHAP should be more considerably investigated because of high frequency of multi-drug resistant pathogens and fatal mortality compared with CAP.

\section{Acknowledgements}

I'm appreciated of Dr. Joon-Seok Ahn, a director of Kangnam hospital in which I did serve my military duty as a public health doctor in the city of Lake, Chuncheon.

\section{References}

1. Carratalà J, Mykietiuk A, Fernández-Sabé N, Suárez C, Dorca J, Verdaguer R, et al. Health care-associated pneumonia requiring hospital admission: epidemiology, antibiotic therapy, and clinical outcomes. Arch Intern Med 2007;167:1393-9.

2. Kollef MH, Shorr A, Tabak YP, Gupta V, Liu LZ, Johannes RS. Epidemiology and outcomes of healthcare-associated pneumonia: results from a large US database of culture-positive pneumonia. Chest 2005;128: 3854-62.

3. Division of Senior Policy in the Office of Aging Society and Population Policy. Current status of the long-term care facilities of the elderly. Seoul: Ministry of Health and Welfare, Republic of Korea; 2008.

4. American Thoracic Society; Infectious Diseases Society of America. Guidelines for the management of adults with hospital-acquired, ventilator-associated, and healthcare-associated pneumonia. Am J Respir Crit Care Med 2005;171:388-416.
5. Polverino E, Dambrava P, Cillóniz C, Balasso V, Marcos MA, Esquinas C, et al. Nursing home-acquired pneumonia: a 10 year single-centre experience. Thorax 2010;65:354-9.

6. Janssens JP, Krause KH. Pneumonia in the very old. Lancet Infect Dis 2004;4:112-24.

7. Ewig S, Welte T, Chastre J, Torres A. Rethinking the concepts of community-acquired and health-care-associated pneumonia. Lancet Infect Dis 2010;10:279-87.

8. Micek ST, Kollef KE, Reichley RM, Roubinian N, Kollef MH. Health care-associated pneumonia and community-acquired pneumonia: a single-center experience. Antimicrob Agents Chemother 2007;51:3568-73.

9. Carratalà J, Fernández-Sabé N, Ortega L, Castellsagué X, Rosón B, Dorca J, et al. Outpatient care compared with hospitalization for community-acquired pneumonia: a randomized trial in low-risk patients. Ann Intern Med 2005;142:165-72.

10. Lim WS, Macfarlane JT. A prospective comparison of nursing home acquired pneumonia with community acquired pneumonia. Eur Respir J 2001;18:362-8.

11. Oken MM, Creech RH, Tormey DC, Horton J, Davis TE, McFadden ET, et al. Toxicity and response criteria of the Eastern Cooperative Oncology Group. Am J Clin Oncol 1982;5:649-55.

12. Lim WS, van der Eerden MM, Laing R, Boersma WG, Karalus N, Town GI, et al. Defining community acquired pneumonia severity on presentation to hospital: an international derivation and validation study. Thorax 2003;58:377-82.

13. Yoon WK, Kim M, Kim YY, Lee YJ, Hwangbo Y, Choi $\mathrm{KJ}$, et al. The clinical and microbial characteristics of healthcare-associated pneumonia. Korean J Med 2010; 78:709-16.

14. Committee for the Japanese Respiratory Society Guidelines in Management of Respiratory. Appendix I: nursing-home acquired pneumonia. Respirology 2004;9 Suppl 1:S51-5.

15. Johnson JC, Jayadevappa R, Baccash PD, Taylor L. Nonspecific presentation of pneumonia in hospitalized older people: age effect or dementia? J Am Geriatr Soc 2000;48:1316-20.

16. Marik PE, Kaplan D. Aspiration pneumonia and dysphagia in the elderly. Chest 2003;124:328-36.

17. Quagliarello V, Ginter S, Han L, Van Ness P, Allore H, Tinetti M. Modifiable risk factors for nursing home-acquired pneumonia. Clin Infect Dis 2005;40:1-6.

18. Shanley C, O'Loughlin G. Dysphagia among nursing home residents: an assessment and management pro- 
YJ Cho et al: Comparative study of nursing home-acquired pneumonia

tocol. J Gerontol Nurs 2000;26:35-48.

19. Falcone M, Serra P, Licata G, Venditti M; Italian Society of Internal Medicine (SIMI). Health care associated pneumonia: a new clinical entity. Arch Intern Med 2008;168:109-10.

20. Mylotte JM. Nursing home-associated pneumonia. Clin Geriatr Med 2007;23:553-65, vi-vii.

21. Kaplan V, Angus DC, Griffin MF, Clermont G, Scott Watson R, Linde-Zwirble WT. Hospitalized community-acquired pneumonia in the elderly: age- and sex-related patterns of care and outcome in the United States. Am J Respir Crit Care Med 2002;165:766-72.
22. Niederman MS, Brito V. Pneumonia in the older patient. Clin Chest Med 2007;28:751-71, vi.

23. Loeb M, Carusone SC, Goeree R, Walter SD, Brazil K, Krueger P, et al. Effect of a clinical pathway to reduce hospitalizations in nursing home residents with pneumonia: a randomized controlled trial. JAMA 2006;295: 2503-10.

24. El Solh AA, Pietrantoni C, Bhat A, Bhora M, Berbary E. Indicators of potentially drug-resistant bacteria in severe nursing home-acquired pneumonia. Clin Infect Dis 2004;39:474-80. 